\title{
Arterial Structure and Function in Ambulatory Adolescents with Cerebral Palsy Are Not Different from Healthy Controls
}

\author{
Audra A. Martin, ${ }^{1}$ Lisa M. Cotie, ${ }^{1}$ Brian W. Timmons, ${ }^{2}$ \\ Jan Willem Gorter, ${ }^{3}$ and Maureen J. MacDonald ${ }^{1}$ \\ ${ }^{1}$ Department of Kinesiology, McMaster University, 1280 Main Street West, Hamilton, ON, Canada L8S 4 K1 \\ ${ }^{2}$ Child Health \& Exercise Medicine Program, McMaster University, 565 Sanatorium Road, Hamilton, ON, Canada L8N $3 Z 5$ \\ ${ }^{3}$ CanChild Centre for Childhood Disability Research, McMaster University, 1400 Main Street West, Hamilton, ON, Canada L8S 1 C7
}

Correspondence should be addressed to Maureen J. MacDonald, macdonmj@mcmaster.ca

Received 1 December 2011; Revised 14 March 2012; Accepted 30 March 2012

Academic Editor: Margaret E. O’Neil

Copyright (c) 2012 Audra A. Martin et al. This is an open access article distributed under the Creative Commons Attribution License, which permits unrestricted use, distribution, and reproduction in any medium, provided the original work is properly cited.

\begin{abstract}
Physical inactivity in youth with cerebral palsy (CP) places them at increased risk of developing cardiovascular disease. The current study assessed indices of arterial health in adolescents with CP, classified as levels I-II of the Gross Motor Function Classification System (GMFCS) $(n=11$, age $13.2 \pm 2.1 \mathrm{yr}$ ), in comparison to age- and sex-matched controls ( $n=11$, age $12.4 \pm 2.3 \mathrm{yr})$. Groups were similar in anthropometric measurements, resting blood pressures, and heart rates. There were no group differences in brachial flow-mediated dilation $(11.1 \pm 7.8$ versus $6.1 \pm 3.6)$, carotid intima-media thickness $(0.42 \pm 0.04$ versus $0.41 \pm 0.03 \mathrm{~mm})$, and distensibility $(0.008 \pm 0.002$ versus $0.008 \pm 0.002 \mathrm{mmHg})$ or central $(4.3 \pm 0.6$ versus $4.1 \pm 0.9 \mathrm{~m} / \mathrm{s})$ and peripheral pulse wave velocity $(7.1 \pm 1.7$ versus $7.6 \pm 1.1 \mathrm{~m} / \mathrm{s})$; CP versus healthy controls, respectively. Vigorous intensity physical activity (PA) was lower in the CP group (CP: $38 \pm 80$ min versus controls: $196 \pm 174 \mathrm{~min}$ ); groups were similar in light and moderate intensity PA levels. Arterial health of ambulatory youth with $\mathrm{CP}$ is not different from a control group despite lower vigorous PA levels. Similar studies need to examine individuals with more pronounced mobility limitations (GMFCS level III-V).
\end{abstract}

\section{Introduction}

Cerebral Palsy (CP) is defined as a disorder of posture and movement due to a nonprogressive disturbance in the developing fetal or infant brain [1]. CP manifests as limitations in gross motor capacity [2], affecting performance in daily mobility over a lifespan [3]. Youth with CP are less physically active than their typically developing peers $[4,5]$ and show an inverse relationship between functional limitations and social participation [6]. Physical inactivity in youth places them at a greater risk of developing a variety of secondary health complications [7] and is also a major controllable risk factor for cardiovascular disease (CVD) [8] .

It has been suggested that one mechanism by which physical activity (PA) exerts its protective effect on cardiovascular health is through positive effects on the endothelium [9], a single layer of cells responsible for the vasodilator response to increased conduit artery flow. A strong relationship between low levels of PA and endothelial dysfunction has been well documented in children [10], potentially predisposing youth with $\mathrm{CP}$ to an increased risk of endothelial dysfunction. Endothelial dysfunction is considered an early and integral manifestation of atherosclerotic disease, which can be evident in the first decade of life [11]. Endothelial dysfunction is an indicator of preclinical vascular disease and for youth with $\mathrm{CP}$ may act as a marker of early changes in vessel function, indicative of future atherosclerotic risk [12].

Pulse wave velocity (PWV) is a sensitive marker of arterial wall stiffness and subsequent marker of cardiovascular risk [13]. In children, PWV is positively correlated with body mass index (BMI), waist circumference (WC), and percentage body fat and negatively correlated with cardiorespiratory fitness and levels of PA [14]. Carotid artery distensibility and carotid artery intima-media thickness ( $\mathrm{c}$ IMT) are two additional indices of arterial health and their role in the development of CVD is widely accepted [15]. 
Strong relationships between cardiovascular risk factors identified in childhood and adolescence and the progression of atherosclerosis in adulthood are emerging [16]. Consistent, positive effects of habitual PA on vessel health have been demonstrated $[14,17]$. Measuring indices of arterial stiffness and endothelial function are therefore important in this young, clinical population in order to identify changes in vascular health at the earliest stage possible.

To our knowledge, there is no study published assessing vessel health in general or its association with levels of habitual PA in youth with CP. Given the fact that children aged 5 to 7 years with CP have lower PA levels than typically developing peers $[4,5]$ we hypothesize that even the most functional adolescents with CP (GMFCS levels I-II) may have decreased levels of PA and altered arterial function and structure compared to an age- and sex-matched control sample.

\section{Methods}

2.1. Participants. Twenty-two adolescents (9-16 yrs) were recruited; of which, 11 individuals with $\mathrm{CP}$ ( 8 boys; mean \pm SD age of $13.2 \pm 2.1 \mathrm{yr}$ ) were recruited from the Spasticity Clinic and Teen Transition Clinic at McMaster Children's Hospital, Hamilton, Ontario, Canada. Inclusion criteria for the CP group included a classification of either a level I or II (GMFCS-Expanded \& Revised) [18] indicating that all subjects with CP were ambulatory without use of mobility devices (level I $n=7$, level II $n=4$ ). Subjects were chronological age- and sex-matched to a healthy control group with a mean age of $12.4 \pm 2.3$ yr. Control subjects were healthy, with no known cardiovascular or metabolic conditions and studied without specific exclusion criteria. Experimental procedures were explained to participants and their guardians prior to obtaining written and verbal informed consent/assent from the parent/guardian and participant, respectively. Approval from the Hamilton Health Sciences and McMaster University Faculty of Health Sciences Research Ethics Board was obtained for the study.

2.2. Study Design. This study employed a cross-sectional design to characterize the differences in specific measures of vascular structure and function between children with $\mathrm{CP}$ and healthy controls. All measures were noninvasive and took place in a quiet, temperature-controlled room $\left(23^{\circ} \pm\right.$ $1^{\circ} \mathrm{C}$ ) with the participant in a supine position. All subjects were instructed to abstain from vigorous PA 24 hours preand were tested 4 hours postprandialy [19].

2.2.1. Anthropometric Measurements. Sitting and standing height $(\mathrm{cm})$ were measured to the nearest $\mathrm{mm}$ without shoes and in light clothing. Body mass was measured to the nearest $0.1 \mathrm{~kg}$ using a digital scale, and BMI was calculated. WC was measured $4 \mathrm{~cm}$ above the umbilicus at the end of a normal expiration [20]. Two measurements were taken for each variable with a third required if a difference greater than $4 \mathrm{~mm}$ for height and WC and $0.4 \mathrm{~kg}$ for weight [21, 22 ] existed. For height and weight, the average of the two measurements was reported, and the median value was reported if three measurements were obtained [23]. Waistto-height ratio (WHR) was calculated as the WC divided by the height $(\mathrm{cm})$. As a marker of biological maturity, each individuals' age at peak height velocity (APHV) and time from peak height velocity (TPHV) was calculated using a gender specific equation [24].

2.2.2. Resting Heart Rate and Blood Pressure. Testing sessions began with $10 \mathrm{~min}$. of supine rest to ensure representative resting measurements prior to the commencement of the vascular assessment [25]. Continuous heart rate via a singlelead electrocardiograph and brachial blood pressure (BP) measurements via an automated applanation tonometer with oscillometric cuff calibration (model CBM-7000; Colin Medical Instruments, San Antonio, TX) were collected. All signals (including those described below) were acquired simultaneously using a commercially available data acquisition system (Powerlab model ML795, ADInstruments, Colorado Springs, USA) and software program (LabChart 7; ADInstruments Inc., Colorado Springs, CO, USA). At the end of the vascular assessment, four measurements of seated brachial artery pressure were obtained using an automated sphygmomanometer (Dinamap Pro 100, Critikon LCC, Tampa, FL). The first measurement was used for calibration purposes only and the average of the following three measures was reported [26].

\subsection{Vascular Assessment}

2.3.1. Pulse Wave Velocity. Baseline measurements of PWV were acquired through electrocardiography and photoplethysmography. Both central and peripheral PWV (cPWV and pPWV, resp.) were determined from 20 continuous heart cycles using the equation [13]:

$$
\mathrm{PWV}=\frac{D}{\Delta t},
$$

where $D$ is the distance between measurement sites and $\Delta t$ is the pulse transit time. Arterial waveforms at the common carotid, femoral, and dorsalis pedis arteries were collected using photoplethysmograph (PPG) sensors (IR Plethysmograph; Model MLT1020PPG; ADInstruments, Colorado Springs) on the right side of the body. PPG signals were bandpass-filtered $(5-30 \mathrm{~Hz})$ with the lower $(\leq 5 \mathrm{~Hz})$ and higher frequencies $(\geq 30 \mathrm{~Hz})$ removed in order to assist in the detection of the foot of each waveform. The foot of each waveform was identified as the minimum value of the digitally filtered signal [27] and corresponds to the end of diastole, when the steep rise in the wave begins and appears as a sharp inflection of the original signal [28].

Central PTT was determined using the subtraction method [29]. Similarly, cPWV path length was calculated by subtracting the surface distance between the sternal notch and the carotid PPG placement from that of the sternal notch and the femoral PPG placement. Peripheral pulse transit time was determined as the time delay between the arrival of the femoral artery pulse wave and the dorsalis pedis artery pulse wave [19], with the path length measured as the distance between these two sites. Anthropometric measuring 
tape was used to measure the straightline distances between skin sites (sternal notch to the placement of each PPG sensor) along the surface of the body.

\subsubsection{Carotid Distensibility and Intima-Media Thickness.} Direct measurements of carotid distensibility were acquired as previously described [30] using a combination of highresolution, two-dimensional, B-mode ultrasound images (System FiVe; GE Medical Systems, Horten, Norway) and applanation tonometry (model SPT-301; Millar Instruments, Houston, TX, USA). A hand-held tonometer was positioned over the point of greatest pulsation and held in a fixed position for ten consecutive heart cycles while ultrasound images of the left common carotid artery were collected simultaneously. Absolute carotid artery systolic blood pressures were calculated by calibrating the relative values acquired using applanation tonometry to the calibrated brachial artery blood pressures acquired simultaneously [31, 32].

Ultrasound images were stored offline in Digital Image and Communications in Medicine (DICOM) format for later analysis using a semiautomated edge tracking system (AMS (Artery Measurement System) Image and Data Analysis. Tomas Gustavsson, gustav@alumni.chalmers.se) [30]. In each frame, carotid artery (minimum, mean, and maximum) lumen diameters were calculated from roughly 100 measurement markers along the vessel wall within the region of interest (ROI), for a total of 110000 measures in a 10 heart cycle data sample. Distensibility was calculated using the equation [13]:

$$
\text { Distensibility }=\frac{\prod\left(d_{\max } / 2\right)^{2}-\prod\left(d_{\min } / 2\right)^{2}}{\prod\left(d_{\max } / 2\right)^{2} \times \mathrm{PP}},
$$

where $d_{\max }$ is the maximum diameter, $d_{\min }$ is the minimum diameter, and PP is carotid pulse pressure, the change in pressure from DBP and SBP. The mean carotid diameter was calculated using the average of all diameters acquired throughout the ten heart cycles. The same software program and ultrasound images were used on the far wall of the carotid artery for measurement of the ${ }_{c}$ IMT.

2.3.3. Flow-Mediated Dilation Assessment. The flow-mediated dilation (FMD) assessment has been shown to be the most reproducible and least variable of the techniques used to measure endothelial function in children [33]. With the participant in the supine position, the left arm was positioned (roughly $80^{\circ}$ from the torso) and immobilized so that an optimal image of the brachial artery could be obtained in a comfortable position [34]. A sphygmomanometric cuff was placed on the forearm, below the medial epicondyle [35], and remained deflated while baseline data were collected. B-mode ultrasound images of the left brachial artery were collected through two-dimensional grayscale ultrasound imaging using a $10 \mathrm{MHz}$ linear array probe (System FiVe; GE Medical Systems, Horten, Norway). A baseline longitudinal image of the brachial artery ( 3 consecutive cardiac cycles) was acquired by a single ultrasonographer. An intensity-weighted sample volume was attained and the gate width was therefore adjusted accordingly.

To create the flow stimulus, the forearm cuff was instantaneously inflated to a standardized, suprasystolic pressure of $200 \mathrm{mmHg}$ to ensure arterial inflow occlusion and ischemia of downstream vessels and tissue [35]. The cuff was instantaneously deflated after $5 \mathrm{~min}$. of occlusion and the first $30 \mathrm{sec}$. of reactive hyperemic blood velocity signals were collected using pulsed-wave Doppler ultrasound. The forward and reverse frequency signals were processed by an external spectral analysis system (Neurovision $500 \mathrm{M}$, Multigon Ind; Yonkers NY) and an intensity-weighted calculated mean was output into a data acquisition system (Powerlab model ML795). B-mode ultrasound images of the brachial artery over three consecutive heart cycles were stored every $15 \mathrm{sec}$. from $30 \mathrm{sec}$. until $3 \mathrm{~min}$. after cuff.

End-diastolic frames were extracted from each sequence of images using a DICOM editing software program (Sante DICOM Editor 3.1.13, Santesoft, Athens, Greece). The semiautomated edge detection software program (AMS) was used to detect the vessel diameters within a specific ROI for the three end-diastolic frames at each time point. The peak dilation of the vessel was established as the single largest enddiastolic diameter $(\mathrm{mm})$ measured from $30 \mathrm{sec}$. to $3 \mathrm{~min}$. after cuff release. From this data, the absolute FMD ( $\mathrm{mm}$ ) and relative FMD (\%FMD) were calculated as follows [36]:

$$
\begin{aligned}
\text { Absolute FMD }= & \text { Peak Diameter }(\mathrm{mm}) \\
& - \text { Baseline Diameter }(\mathrm{mm}), \\
\text { Relative FMD }= & \left(\frac{\text { Absolute FMD }}{\text { Baseline Diameter }}\right) \times 100 \% .
\end{aligned}
$$

The following equation was used to calculate shear rate (SR) for each participant [37]:

$$
\text { Shear Rate }=8 \times\left(\frac{\text { Velocity }}{\text { Diameter }}\right) \text {, }
$$

where velocity represents the mean blood flow velocity of the velocity profile of the first $30 \mathrm{sec}$. after cuff release and the baseline brachial diameter $(\mathrm{mm})$ is used for the internal artery diameter value. The area under the curve of the shear rate was calculated from the mean of the first point, using the trapezoid rule to obtain the area under the entire curve (GraphPad Prism version 4.00 for Windows, GraphPad Software, San Diego California USA, http://www.graphpad .com/). Relative FMD (\%FMD) was normalized to the area under the entire SR curve and reported as \%FMD/SR $\mathrm{AUC}$ :

$$
\text { Normalized FMD }=\left(\frac{\% \text { FMD }}{S_{\mathrm{AUC}}}\right) .
$$

This method of analysis provides values of absolute maximum dilation $(\mathrm{mm})$, time to reach peak dilation (sec.), and a raw calculation of the SR stimulus $\left(\mathrm{SR}_{\mathrm{AUC}}\right)$.

2.4. Physical Activity. Habitual PA patterns were assessed using the Exercise Questionnaire adopted from Brunton and Bartlett, used in a longitudinal study describing exercise participation of adolescents with CP [38]. This recall 
questionnaire provides information regarding the frequency, duration, and intensity of PA performed in the previous week. This questionnaire, based on the "Previous Day Physical Activity Record" by Weston et al. (1997) [39], was used to assess PA in both the CP and control group.

2.5. Statistical Analysis. Statistical analyses were performed using SPSS Statistics, version 19.0 (SPSS, Inc., Chicago, IL). Data distribution was initially examined for normality using the Shapiro-Wilk's Test and homogeneity of variance using Levene's Test. Independent $t$-tests were used to compare group differences in all vascular indices, anthropometric measures, and levels of PA. Analyses of vascular indices were also completed with chronological age as a covariate. Statistical significance for all analyses was set at $P \leq 0.05$.

\section{Results}

Characteristics of the study population are described in Table 1. The control and CP group were of similar age, height, weight, WC, WHR, and BMI. There were no group differences in resting seated brachial systolic blood pressure, dias-tolic blood pressure, mean arterial pressure, or resting supine heart rate.

Outcomes of the flow-mediated dilation (FMD) assessment are reported in Table 2. It must be noted that one participant with CP was removed from all FMD analysis due to inadequate ultrasound image quality of postocclusion data. One control subject was also identified as an outlier (via box plot and a response greater than 2 SD above the mean) and removed from the analysis. Thus, all statistical analyses of endothelial function (Table 2) were performed with an $n=10$ in each group, with the exception of the preocclusion brachial diameters $(n=11)$ as all pre-occlusion data remained acceptable for analysis. There were no differences between groups $(P>0.05)$ in pre-occlusion brachial diameter $(\mathrm{mm})$ or peak diameter $(\mathrm{mm})$ reached during reactive hyperemia (Table 2). There were no differences in the SR stimulus or time taken to reach peak diameter between groups (Table 2).

There were no differences in baseline measures of carotid distensibility, cIMT, or baseline carotid diameter between groups (Table 3 ). One control subject was a significant outlier and removed from analysis of distensibility (CON, $n=10$ ) and one CP subject could not be included in the analysis of ${ }_{c}$ IMT due to insufficient clarity of the far wall IMT for pro-per identification (CP, $n=10)$. No differences were seen in CPWV or pPWV or PTT between groups (Table 3 ). One individual from the control group could not be included in the analysis due to an arrhythmia that did not permit appropriate analysis of the PWV data (CON, $n=10)$.

The total number of minutes/week of PA in each intensity category is reported in Figure 1(a). There were no group differences in the total number of minutes spent in light and moderate PA. The CP group reported a significantly smaller amount of vigorous PA weekly than the control group (Figure 1(a)) with over $60 \%$ of individuals in the CP group reporting 0 minutes of total time spent performing vigorous
TABLE 1: Subject characteristics.

\begin{tabular}{lccc}
\hline & $\begin{array}{c}\text { Control } \\
(n=11)\end{array}$ & $\begin{array}{c}\text { CP } \\
(n=11)\end{array}$ & P value \\
\hline Age, yrs & $12.4 \pm 2.3$ & $13.2 \pm 2.1$ & 0.458 \\
Height, m & $1.6 \pm 0.1$ & $1.5 \pm 0.1$ & 0.169 \\
Weight, kg & $49.3 \pm 14.2$ & $41.4 \pm 8.4$ & 0.129 \\
APHV & $13.08 \pm 0.9$ & $14.02 \pm 1.3$ & 0.062 \\
TPHV, yrs & $-0.66 \pm 2.1$ & $-0.86 \pm 1.7$ & 0.809 \\
WC, cm & $69.8 \pm 8.8$ & $67.3 \pm 7.2$ & 0.478 \\
WHR & $0.44 \pm 0.05$ & $0.45 \pm 0.06$ & 0.750 \\
BMI, kg/m ${ }^{2}$ & $19.5 \pm 3.7$ & $18.4 \pm 3.2$ & 0.474 \\
BMI percentile & $57 \pm 31$ & $38 \pm 33$ & 0.178 \\
Resting HR, bpm & $68 \pm 10$ & $74 \pm 13$ & 0.278 \\
Resting systolic BP, mmHg & $113 \pm 8$ & $106 \pm 12$ & 0.164 \\
Resting diastolic BP, mmHg & $65 \pm 5$ & $62 \pm 6$ & 0.169 \\
Resting MAP, mmHg & $84 \pm 3$ & $81 \pm 6$ & 0.152 \\
\hline
\end{tabular}

Values are represented as means \pm SD. APHV: age at peak height velocity; TPHV: time to peak height velocity; WC: waist circumference; WHR: waistto-height ratio; BMI: body mass index; HR: heart rate; BPM: beats per minute; BP: blood pressure; MAP: mean arterial pressure.

PA in the previous week. Furthermore, when total PA time/week was calculated (combining each intensity of PA), there were no significant differences between groups (CP: $4260 \mathrm{~min} /$ week versus Controls: $4840 \mathrm{~min} /$ week) (Figure 1(b)).

\section{Discussion}

Over time, decreased levels of PA are generally associated with impairments of vascular function and structure and increased cardiovascular risk. This becomes particularly important when PA levels are limited in children and adolescents with a physical disability, such as cerebral palsy. Thus, early vascular assessments in this at-risk population may assist in determining potential CV risk factors. In this study we purposefully studied vascular health in the most functional adolescents with CP to contrast their PA levels and vascular health with their healthy peers. The primary findings did not confirm our hypothesis that arterial function and structure in adolescents with CP (GMFCS level I-II) are different from a healthy control group despite individuals with CP spending significantly less time performing vigorous PA in comparison to their typically developing peers.

In this study, the primary risk factor (for future cardiovascular health) of interest was level of PA, as measured using the Exercise Questionnaire [38]. Both groups spent similar amounts of time performing light-to-moderate PA; however, the CP group spent a significantly less amount of time engaging in vigorous intensity PA. Despite this discrepancy in time spent in high intensity PA, no group differences were seen in any of the measured indices of vascular health. It has been suggested that the strongest relationships between exercise interventions (comparable to levels of PA) and enhanced endothelial function exist in groups with relatively impaired FMD a priori. The tightest correlations between PA and 
TABLE 2: Group comparisons of brachial vascular dimensions and FMD response.

\begin{tabular}{|c|c|c|c|}
\hline & Control $(n=10)$ & $\mathrm{CP}(n=10)$ & $P$ value \\
\hline Preocclusion diameter, $\mathrm{mm}$ & $3.20 \pm 0.37$ & $3.08 \pm 0.48$ & 0.803 \\
\hline RH peak diameter, mm & $3.40 \pm 0.39$ & $3.48 \pm 0.38$ & 0.815 \\
\hline Absolute FMD, mm & $0.19 \pm 0.11$ & $0.33 \pm 0.21$ & 0.075 \\
\hline Relative FMD (\%FMD) & $6.1 \pm 3.6$ & $11.1 \pm 7.8$ & 0.080 \\
\hline Normalized (\%FMD/SR $\left.{ }_{\text {AUC }}\right)$ & $0.0027 \pm 0.0015$ & $0.0046 \pm 0.0033$ & 0.126 \\
\hline Mean SR & $530 \pm 250$ & $544 \pm 198$ & 0.788 \\
\hline Time to peak, $s$ & $110 \pm 45$ & $102 \pm 46$ & 0.826 \\
\hline $\mathrm{PP}, \mathrm{mmHg}$ & $48 \pm 10$ & $45 \pm 13$ & 0.523 \\
\hline
\end{tabular}

Values are represented as means \pm SD. RH: reactive hyperemia; FMD: flow mediated dilation; SR: shear rate; SR $\mathrm{AUC}_{\mathrm{U}}$ shear rate area under the curve. Note. $n=11$ in both groups for baseline brachial diameter.

TAвLE 3: Group comparisons of PTT, PWV, carotid vascular dimensions, and carotid distensibility.

\begin{tabular}{lccc}
\hline & Control $(n=10)$ & CP $(n=11)$ & $P$ value \\
\hline Central PTT & $0.103 \pm 0.032$ & $0.089 \pm 0.013$ & $4.3 \pm 0.6$ \\
cPWV (m/s) & $4.1 \pm 0.9$ & $0.111 \pm 0.028$ & 0.454 \\
Peripheral PTT & $0.108 \pm 0.012$ & $7.1 \pm 1.7$ & 0.977 \\
pPWV (m/s) & $7.6 \pm 1.1$ & $5.63 \pm 0.74$ & 0.768 \\
Baseline diameter, mm & $5.73 \pm 0.29$ & $0.42 \pm 0.04$ & 0.450 \\
cIMT, mm & $0.41 \pm 0.03$ & $0.077 \pm 0.012$ & 0.690 \\
Wall/lumen ratio & $0.072 \pm 0.007$ & $0.008 \pm 0.002$ & 0.576 \\
Distensibility, $\mathrm{mmHg}^{-1}$ & $0.008 \pm 0.002$ & $0.17 \pm 0.06$ & 0.832 \\
Compliance, $\mathrm{mm}^{2} / \mathrm{mmHg}^{-}$ & $0.19 \pm 0.03$ & $42 \pm 11$ & 0.474 \\
PP (mmHg) & $36 \pm 10$ & 0.208 \\
\hline
\end{tabular}

Values are represented as means \pm SD. PTT: pulse transit time; PWV: pulse wave velocity, distensibility and compliance: Control, $n=10$; IMT: intima-media thickness and wall/lumen ratio: $\mathrm{CP}, n=10$; PP: pulse pressure.

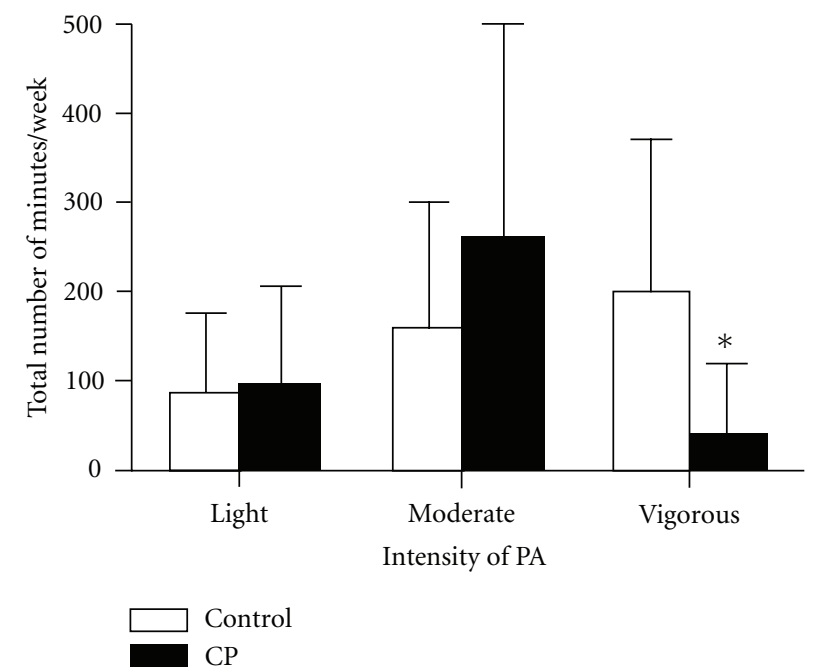

(a)

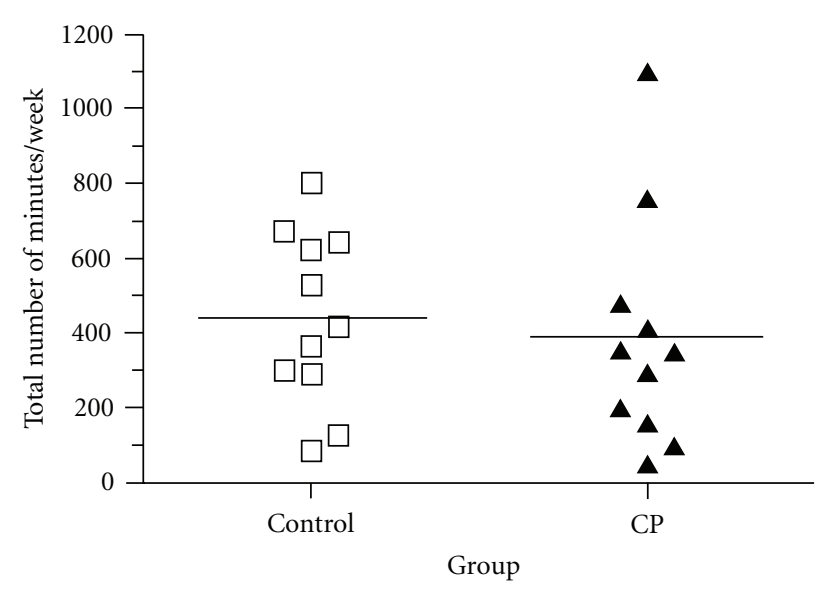

(b)

Figure 1: (a) Group comparisons of weekly PA according to intensity. (b) Group comparisons of total PA (summation of all three intensities) performed in one week, individual data and group means presented; $n=11$ in each group. 
FMD response have been shown to exist in the lowest tertiles of endothelial function [40]. Considering this, there is no reason to believe that the control group has experienced vascular dysfunction, which would predispose them to a positive vascular adaptation as a result of their higher levels of vigorous activity in comparison to the seemingly healthy CP group.

No significant differences between groups were found in cPWV or pPWV. These values were comparable to a previous study assessing PWV in a slightly younger group of healthy children $(10.1 \pm 0.3$ yrs $)$ who showed very similar cPWV values $(4.2 \pm 0.4 \mathrm{~m} / \mathrm{s})$ [14] to those in both groups in the current study (Table 3 ). This indicates preserved arterial stiffness at this time point for both the control and CP group. Similarities in PWV between groups in this study may be reflective of similar levels of low intensity PA, as indicated by the same amount of time spent in light and moderate intensity $\mathrm{PA}$ as well as the same total time spent performing PA per week (Figure 1(b)).

No differences were found between groups in either carotid distensibility or ${ }_{c}$ IMT. Throughout the lifespan, habitual PA has been shown to positively influence arterial distensibility $[14,18]$. Age-related decreases in arterial distensibility and increases in stiffness have been reported [18]; however, increased levels of PA have been suggested to delay the agedependent loss of arterial distensibility, in proportion to the amount and/or intensity of exercise [18, 41, 42]. Although there was no difference in distensibility between the CP and control group at this time, sufficient rationale is provided for this clinical group of adolescents to increase their levels of high intensity PA at an early stage and maintain these behaviours into adulthood in an attempt to mitigate these normative age-related changes.

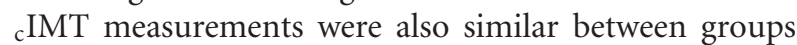
and were comparable to other control groups used in previous studies [43, 44]. Iannuzzi and colleagues (2004) [43] characterized the differences in ${ }_{c}$ IMT between obese children and age-matched control subjects (6-14 years) and showed a significantly greater IMT in the obese group in comparison to the healthy controls $(0.55 \pm 0.08 \mathrm{~mm}$ versus $0.49 \pm$ $0.09 \mathrm{~mm}$ ). The ${ }_{\mathrm{c}} \mathrm{IMT}$ of the obese children in the aforementioned study was approximately $24 \%$ and $25 \%$ greater than the ${ }_{\mathrm{c}}$ IMT of the present study's CP and control group, suggesting healthy vascular structure in both groups in the current study.

In a previous study assessing the relationship between habitual PA (as measured using the double labeled water approach) and brachial FMD in 5-10-year-old children, a significant correlation was found $(r=0.39, P=0.007)$, highlighting PA as the most influential variable in predicting the FMD response [9]. This group reported that physical fitness, as assessed using an incremental discontinuous treadmill-based exercise test, and levels of PA, as measured using Actigraph accelerometers, were lowest in the lowest \%FMD and $\% \mathrm{FMD} / \mathrm{SR}_{\mathrm{AUC}}$ tertile. These relationships between fitness, PA, and FMD response were significant, and it was concluded that PA measurements were the best predictors of endothelial (dys) function in this young group [40]. These data support the concept that PA exerts its protective effect on CV health via the endothelium and draws attention to the role of lifestyle modifications, specifically increases in levels of habitual PA in pediatric practice.

This cross-sectional study is the first to characterize indices of vascular health in higher functioning youth with $\mathrm{CP}$ and to make comparisons to a group of their typically developing peers. Children harbouring classic CV risk factors, including physical inactivity have been shown to exhibit impairments in vascular function and structure early in life and have an increased risk of premature atherosclerosis in adulthood [44]. It has been shown that levels of both PA and inactivity track significantly from adolescence ( 9 to $18 \mathrm{yrs}$ ) to young adulthood placing inactive children at an increased risk of becoming physically sedentary adults [45]. With evidence of physical inactivity being a significant precursor to CVD-related death and moderate levels of fitness providing protective effects against the influence of traditional risk factors on mortality [46], the value of well-established, healthy patterns of habitual PA in pediatric practice must not be overlooked. In a group of youth that may have increased susceptibility to physical inactivity, identifying any early alterations in vascular function and structure may assist in identifying preclinical vascular disease, allowing for intervention at the earliest stage possible.

\section{Limitations}

The FMD methodology used in the current study is relatively straightforward and noninvasive. However, limitations to the procedure are present. It is possible that during the FMD assessment peak dilation was underestimated as images were taken every $15 \mathrm{sec}$. for three heart cycles and not continuously for $3 \mathrm{~min}$. following cuff release. This is a limitation of the storing capabilities of the equipment used; thus we chose to collect diameter data at fifteen-second intervals to attempt to represent the complete diameter profile following cuff release. The current results are limited to highly functioning, ambulatory individuals with CP and their typically developing peers. It is difficult to say if these results are applicable to prepubertal or postpubertal individuals as it can be assumed a mixed sample was represented. In this investigation, we did not control for or assess diet, vitamin ingestion or bloodborne CVD markers and therefore we cannot account for the contribution of these factors in any changes in vascular function.

One possible explanation for our finding of similar vascular structure and function despite differences in the amount of vigorous PA is that light-to-moderate PA is the main determinant of vascular health and that vigorous exercise is not necessary to maintain normal vascular structure and function in youth. It is also possible that the method used in the current study to assess PA (CP specific questionnaire) was not sensitive enough to determine absolute differences at all intensities or that confounding factors might result in a relative underestimation of the vigorous component of exercise for youth with CP. Future studies, which include more direct measurement of activity levels, may delineate the relationship between absolute activity levels and arterial health. 


\section{Conclusion}

Although no differences in vascular structure or function between youth (9-16 years) with CP and typically developing peers were observed in the current study, the establishment of techniques to assess arterial health in youth with CP is critically important for determining future CV risk in this clinical population. This study confirms the feasibility of the use of these vascular assessment techniques in this population and presents potential for future, longitudinal assessments of individuals with CP across all levels of GMFCS classification. Each measurement of cardiovascular health was well tolerated and widely accepted by both participants and their parent/guardian(s). The consequences of significantly decreased amounts of time spent in vigorous PA for youth with CP, at this time and potentially into adulthood, remain unknown. For future research it is of interest to assess whether vessel health is compromised in youth and adults with more pronounced decreased levels of PA such as those in GMFCS levels III-V. Identifying these parameters may act as a tool for risk stratification in this population, thereby permitting identification of children who would benefit most from intensified PA and/or exercise interventions.

\section{Acknowledgments}

This paper is based on a master thesis in kinesiology at McMaster University written by the first author and funded by NSERC. This study was done as part of the Stay-FIT study that is funded by the Ontario Federation for Cerebral Palsy. Special thanks are due to the participants and their families for their valuable time and effort with this study.

\section{References}

[1] P. Rosenbaum, N. Paneth, A. Leviton et al., "A report: the definition and classification of cerebral palsy," Developmental Medicine and Child Neurology, vol. 109, pp. 8-14, 2007.

[2] P. L. Rosenbaum, S. D. Walter, S. E. Hanna et al., "Prognosis for gross motor function in cerebral palsy: creation of motor development curves," Journal of the American Medical Association, vol. 288, no. 11, pp. 1357-1363, 2002.

[3] J. W. Gorter, "Rehabilitative therapies for the child with cerebral palsy: focus on family, function and fitness," Minerva Pediatrica, vol. 61, no. 4, pp. 425-440, 2009.

[4] K. F. Bjornson, B. Belza, D. Kartin, R. Logsdon, and J. F. McLaughlin, "Ambulatory physical activity performance in youth with cerebral palsy and youth who are developing typically," Physical Therapy, vol. 87, no. 3, pp. 248-257, 2007.

[5] C. A. Maher, M. T. Williams, T. Olds, and A. E. Lane, "Physical and sedentary activity in adolescents with cerebral palsy," Developmental Medicine and Child Neurology, vol. 49, no. 6, pp. 450-457, 2007.

[6] R. Schenker, W. Coster, and S. Parush, "Participation and activity performance of students with cerebral palsy within the school environment," Disability and Rehabilitation, vol. 27, no. 10, pp. 539-552, 2005.

[7] M. J. LaMonte, S. N. Blair, and T. S. Church, "Physical activity and diabetes prevention," Journal of Applied Physiology, vol. 99, no. 3, pp. 1205-1213, 2005.
[8] S. N. Blair, H. W. Kohl, C. E. Barlow, R. S. Paffenbarger, L. W. Gibbons, and C. A. Macera, "Changes in physical fitness and all-cause mortality: a prospective study of healthy and unhealthy men," Journal of the American Medical Association, vol. 273, no. 14, pp. 1093-1098, 1995.

[9] R. A. Abbott, M. A. Harkness, and P. S. W. Davies, "Correlation of habitual physical activity levels with flow-mediated dilation of the brachial artery in 5-10 year old children," Atherosclerosis, vol. 160, no. 1, pp. 233-239, 2002.

[10] K. Pahkala, O. J. Heinonen, H. Lagström et al., "Vascular endothelial function and leisure-time physical activity in adolescents," Circulation, vol. 118, no. 23, pp. 2353-2359, 2008.

[11] H. C. Stary, "Evolution and progression of atherosclerotic lesions in coronary arteries of children and young adults," Arteriosclerosis, vol. 9, supplement 1, pp. I19-I32, 1989.

[12] J. A. Vita and J. F. Keaney, "Endothelial function: a barometer for cardiovascular risk?” Circulation, vol. 106, no. 6, pp. 640642, 2002.

[13] M. F. O’Rourke, J. A. Staessen, C. Vlachopoulos, D. Duprez, and G. E. Plante, "Clinical applications of arterial stiffness; definitions and reference values," American Journal of Hypertension, vol. 15, no. 5, pp. 426-444, 2002.

[14] S. Sakuragi, K. Abhayaratna, K. J. Gravenmaker et al., "Influence of adiposity and physical activity on arterial stiffness in healthy children the lifestyle of our kids study," Hypertension, vol. 53, no. 4, pp. 611-616, 2009.

[15] D. H. O’Leary, J. F. Polak, R. A. Kronmal, T. A. Manolio, G. L. Burke, and S. K. Wolfson, "Carotid-artery intima and media thickness as a risk factor for myocardial infarction and stroke in older adults," New England Journal of Medicine, vol. 340, no. 1, pp. 14-22, 1999.

[16] O. T. Raitakari, M. Juonala, M. Kähönen et al., "Cardiovascular risk factors in childhood and carotid artery intima-media thickness in adulthood: the cardiovascular risk in young finns study," Journal of the American Medical Association, vol. 290, no. 17, pp. 2277-2283, 2003.

[17] D. R. Seals, C. A. DeSouza, A. J. Donato, and H. Tanaka, "Habitual exercise and arterial aging," Journal of Applied Physiology, vol. 105, no. 4, pp. 1323-1332, 2008.

[18] J. W. Gorter, J. Slaman, D. Bartlett, and H. J. G. Van den BergEmons, "Reliability of the gross motor function classification system expanded and revised (GMFCS-ER) when used with adolescents and young adults with cerebral palsy," Developmental Medicine \& Child Neurology, vol. 53, supplement 5, pp. 42-43, 2011.

[19] M. Rakobowchuk, M. I. Stuckey, P. J. Millar, L. Gurr, and M. J. MacDonald, "Effect of acute sprint interval exercise on central and peripheral artery distensibility in young healthy males," European Journal of Applied Physiology, vol. 105, no. 5, pp. 787-795, 2009.

[20] M. C. J. Rudolf, J. Walker, and T. J. Cole, "What is the best way to measure waist circumference?" International Journal of Pediatric Obesity, vol. 2, no. 1, pp. 58-61, 2007.

[21] D. A. Bailey, "The Saskatchewan pediatric bone mineral accrual study: bone mineral acquisition during the growing years," International Journal of Sports Medicine, vol. 18, supplement 3, pp. S191-S194, 1997.

[22] L. B. Sherar, R. L. Mirwald, A. D. G. Baxter-Jones, and M. Thomis, "Prediction of adult height using maturity-based cumulative height velocity curves," Journal of Pediatrics, vol. 147, no. 4, pp. 508-514, 2005.

[23] W. Ross and M. Marfell-Jones, "Kinanthropometry," in Physiological Testing of the High-Performance Athlete, pp. 223-308, Human Kinetics Books, Champaign, Ill, USA, 1991. 
[24] R. L. Mirwald, A. D. G. Baxter-Jones, D. A. Bailey, and G. P. Beunen, "An assessment of maturity from anthropometric measurements," Medicine and Science in Sports and Exercise, vol. 34, no. 4, pp. 689-694, 2002.

[25] R. A. Harris, S. K. Nishiyama, D. W. Wray, and R. S. Richardson, "Ultrasound assessment of flow-mediated dilation," $\mathrm{Hy}$ pertension, vol. 55, no. 5, pp. 1075-1085, 2010.

[26] T. G. Pickering, J. E. Hall, L. J. Appel et al., "Recommendations for blood pressure measurement in humans: an AHA scientific statement from the Council on High Blood Pressure Research Professional and Public Education Subcommittee.," Journal of Clinical Hypertension, vol. 7, no. 2, pp. 102-109, 2005.

[27] R. Munakata, H. Katakai, T. Ueki, J. Kurosaka, K. I. Takao, and K. I. Tadano, "Total synthesis of (+)-macquarimicin A," Journal of the American Chemical Society, vol. 125, no. 48, pp. 14722-14723, 2003.

[28] W. W. Nichols and M. F. O'Rourke, McDonald's Blood Flow in Arteries: Theoretical, Experimental and Clinical Principles, A Hodder Arnold Publication, 5th edition, 2005.

[29] T. Weber, M. Ammer, M. Rammer et al., "Noninvasive determination of carotid-femoral pulse wave velocity depends critically on assessment of travel distance: a comparison with invasive measurement," Journal of Hypertension, vol. 27, no. 8, pp. 1624-1630, 2009.

[30] K. D. Currie, N. A. Proudfoot, B. W. Timmons, and M. J. MacDonald, "Noninvasive measures of vascular health are reliable in preschool-aged children," Applied Physiology, Nutrition and Metabolism, vol. 35, no. 4, pp. 512-517, 2010.

[31] R. Kelly and D. Fitchett, "Noninvasive determination of aortic input impedance and external left ventricular power output: a validation and repeatability study of a new technique," Journal of the American College of Cardiology, vol. 20, no. 4, pp. 952963, 1992.

[32] B. Fernhall and S. Agiovlasitis, "Arterial function in youth: window into cardiovascular risk," Journal of Applied Physiology, vol. 105, no. 1, pp. 325-333, 2008.

[33] A. E. Donald, M. Charakida, T. J. Cole et al., "Non-invasive assessment of endothelial function. Which technique?" Journal of the American College of Cardiology, vol. 48, no. 9, pp. 1846-1850, 2006.

[34] G. Atkinson, A. M. Batterham, M. A. Black et al., "Is the ratio of flow-mediated dilation and shear rate a statistically sound approach to normalization in cross-sectional studies on endothelial function?" Journal of Applied Physiology, vol. 107, no. 6, pp. 1893-1899, 2009.

[35] M. C. Corretti, T. J. Anderson, E. J. Benjamin et al., "Guidelines for the ultrasound assessment of endothelial-dependent flow-mediated vasodilation of the brachial artery: a report of the international brachial artery reactivity task force," Journal of the American College of Cardiology, vol. 39, no. 2, pp. 257265, 2002.

[36] T. J. Anderson, A. Uehata, M. D. Gerhard et al., "Close relation of endothelial function in the human coronary and peripheral circulations," Journal of the American College of Cardiology, vol. 26, no. 5, pp. 1235-1241, 1995.

[37] B. A. Parker, T. L. Trehearn, and J. R. Meendering, "Pick your Poiseuille: normalizing the shear stimulus in studies of flowmediated dilation," Journal of Applied Physiology, vol. 107, no. 4, pp. 1357-1359, 2009.

[38] L. K. Brunton and D. J. Bartlett, "Description of exercise participation of adolescents with cerebral palsy across a 4-year period," Pediatric Physical Therapy, vol. 22, no. 2, pp. 180-187, 2010 .
[39] A. T. Weston, R. Petosa, and R. R. Pate, "Validation of an instrument for measurement of physical activity in youth," Medicine and Science in Sports and Exercise, vol. 29, no. 1, pp. 138-143, 1997.

[40] N. D. Hopkins, G. Stratton, T. M. Tinken et al., "Relationships between measures of fitness, physical activity, body composition and vascular function in children," Atherosclerosis, vol. 204, no. 1, pp. 244-249, 2009.

[41] T. Kakiyama, M. Matsuda, and S. Koseki, "Effect of physical activity on the distensibility of the aortic wall in healthy males," Angiology, vol. 49, no. 9, pp. 749-757, 1998.

[42] D. R. Seals, A. E. Walker, G. L. Pierce, and L. A. Lesniewski, "Habitual exercise and vascular ageing," Journal of Physiology, vol. 587, no. 23, pp. 5541-5549, 2009.

[43] A. Iannuzzi, M. R. Licenziati, C. Acampora et al., "Increased carotid intima-media thickness and stiffness in obese children," Diabetes Care, vol. 27, no. 10, pp. 2506-2508, 2004.

[44] B. Hacihamdioğlu, V. Okutan, Y. Yozgat et al., "Abdominal obesity is an independent risk factor for increased carotid intima- media thickness in obese children," Turkish Journal of Pediatrics, vol. 53, no. 1, pp. 48-54, 2011.

[45] M. Juonala, J. S. A. Viikari, M. Kähönen et al., "Life-time risk factors and progression of carotid atherosclerosis in young adults: the Cardiovascular Risk in Young Finns study," European Heart Journal, vol. 31, no. 14, pp. 1745-1751, 2010.

[46] R. Telama, X. Yang, J. Viikari, I. Välimäki, O. Wanne, and O. Raitakari, "Physical activity from childhood to adulthood: a 21-year tracking study," American Journal of Preventive Medicine, vol. 28, no. 3, pp. 267-273, 2005. 


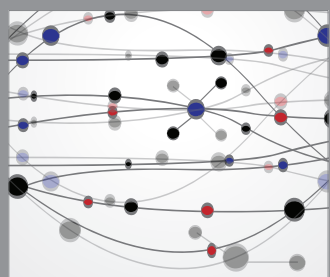

The Scientific World Journal
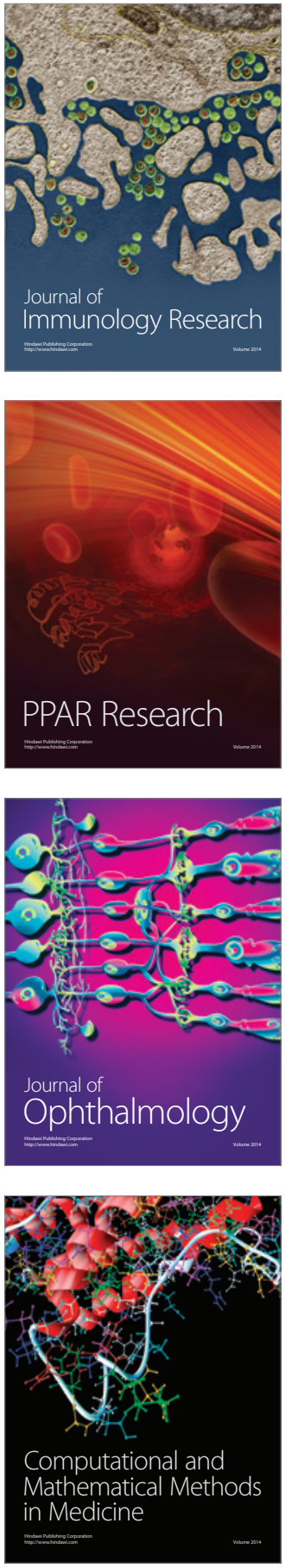

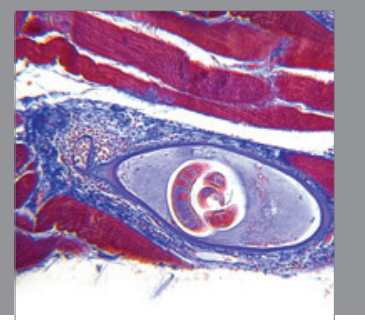

Gastroenterology

Research and Practice
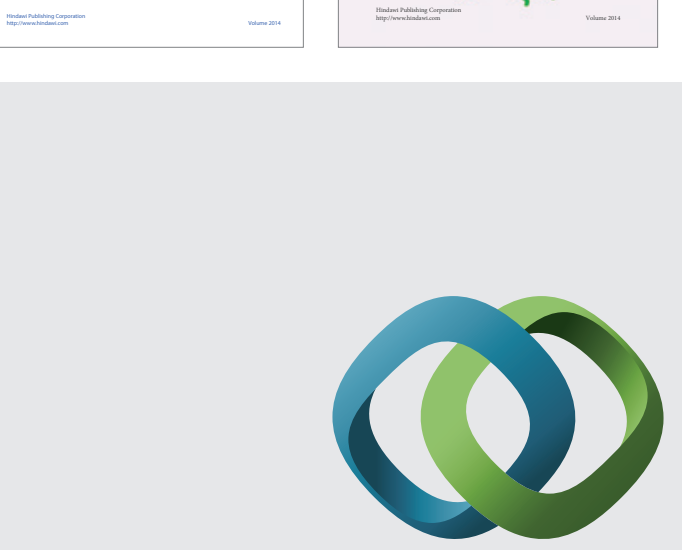

\section{Hindawi}

Submit your manuscripts at

http://www.hindawi.com
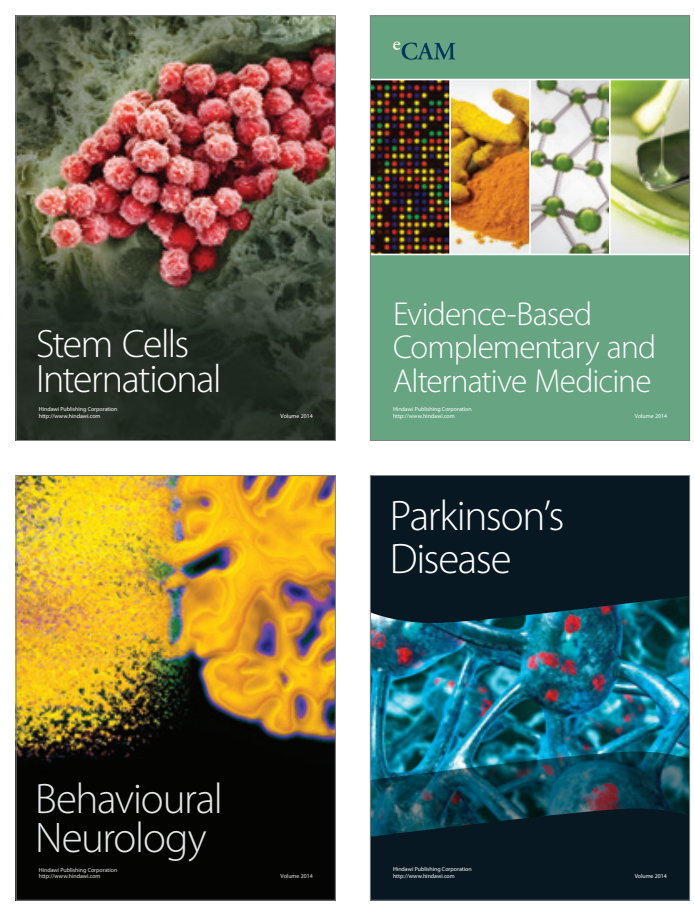

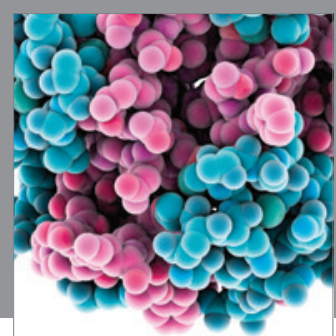

Journal of
Diabetes Research

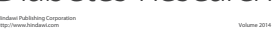

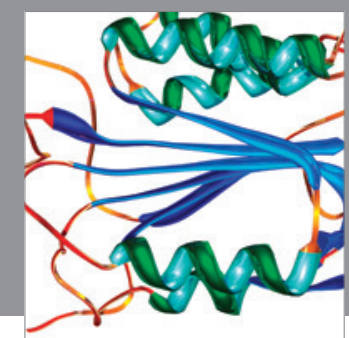

Disease Markers
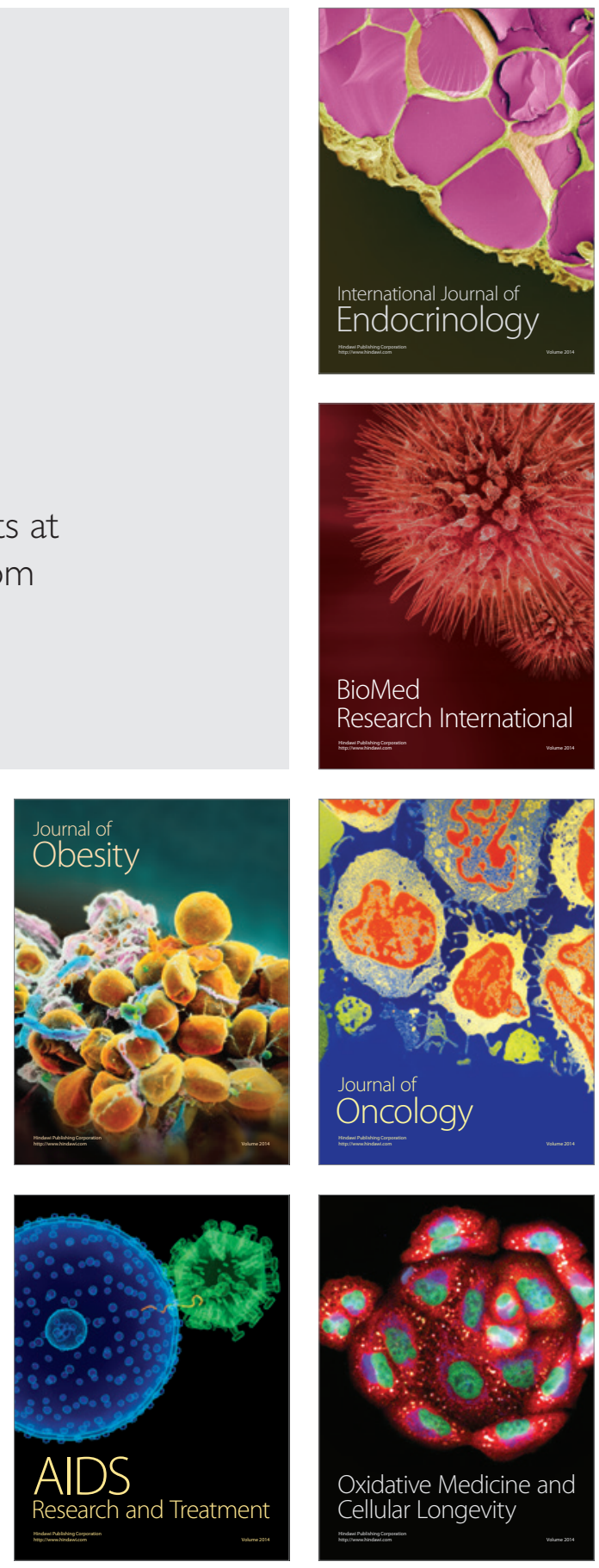